\title{
Criminologie
}

\section{"Alternatives » à la prison : diffusion ou décroissance du contrôle social : une entrevue avec Michel Foucault}

Jean-Paul Brodeur

Volume 26, numéro 1, 1993

Michel Foucault et la (post) modernité

URI : https://id.erudit.org/iderudit/017328ar

DOI : https://doi.org/10.7202/017328ar

Aller au sommaire du numéro

Éditeur(s)

Les Presses de l'Université de Montréal

ISSN

0316-0041 (imprimé)

1492-1367 (numérique)

Découvrir la revue

Citer ce document

Brodeur, J.-P. (1993). « Alternatives » à la prison : diffusion ou décroissance du contrôle social : une entrevue avec Michel Foucault. Criminologie, 26(1), 13-34. https://doi.org/10.7202/017328ar d'utilisation que vous pouvez consulter en ligne. 


\section{ALTERNATIVES À LA PRISON: DIFFUSION OU DÉCROISSANCE DU CONTRÔLE SOCIAL ${ }^{1}$ \\ Une entrevue avec Michel Foucault}

Inutile de vous dire que je suis content d'être ici: mais inutile en même temps de vous dire comment je suis embarrassé - j'ai été embarrassé lorsqu'on m'a appris qu'il fallait que je parle des alternatives à la prison et que j'en parle dans le cadre d'une semaine qui était consacrée à la faillite de la prison.

J'étais embarrassé pour deux raisons: d'abord à cause de ce problème de l'alternative, et ensuite à cause de ce problème de la faillite.

Alternative à la prison; quand on me parle de ça, j'ai immédiatement une réaction enfantine. Je me fais l'impression d'un enfant de 7 ans à qui l'on dit: «Écoute, puisque de toute façon tu vas être puni, qu'est-ce que tu préfères, le fouet ou être privé de dessert ?»

Il me semble que la question de l'alternative à la prison est typiquement de ce genre. Question fausse ou en tout cas question partielle, puisqu'il s'agit en somme de dire aux gens: «Écoutez. Voilà: En admettant le régime actuel de la pénalité, en admettant que vous êtes punis par telle personne ou telle chose, comment pensez-vous que ce système de pénalité doit être mis en œuvre? Est-ce qu'il sera mieux mis en œuvre par la prison ou par un autre type de punition?»

1. Cette conférence-entrevue, a été présentée par Michel Foucault à l'Université de Montréal, le 15 mars 1976, à partir de questions préparées par les organisateurs de cette conférence. Cette activité s'est déroulée dans le cadre d'une semaine sur les droits des détenus. La présentation verbale de Michel Foucault a été enregistrée sur ruban et retranscrite après son départ, avec sa permission. La transcription a été déposée par les organisateurs de cette conférence dans un classeur de l'Université de Montréal, où nous l'avons retrouvée il y a peu de temps. Ce texte n'a donc pas été revu par Michel Foucault lui-mêrne, mais par Jean-Paul Brodeur. Nous avons tenté dans toute la mesure du possible de respecter la lettre de la transcription originale et de conserver le style oral et animé de la présentation. La très grande majorité des corrections ont eu pour objet le rétablissement de la ponctuation, la suppression des répétitions de mots dans la même phrase et des changements - peu fréquents - dans l'ordre des mots. En effet, le discours oral a ceci de particulier qu'il place les compléments d'objet en début de phrase, avant le verbe. Le discours écrit répugne parfois à ces inversions, qui rendent la phrase inintelligible lorsqu'elles s'accumulent. Le lecteur du discours écrit ne dispose pas, comme l'auditeur d'une conférence, des inflexions de voix du conférencier pour scander la phrase et l'interpréter selon son sens. En aucun cas, n'avons-nous ajouté une phrase, un membre de phrase ou même un mot. 
Si bien que je crois qu'à la question de l'alternative à la prison, il faut répondre par un premier scrupule, par un premier doute ou par un premier éclat de rire, comme vous voudrez; et si nous ne voulions pas être punis par ceux-là, ou pour ces raisons-là... et si nous ne voulions pas être punis du tout? Et si, après tout, nous n'étions pas capables de savoir réellement ce que veut dire punir?

Est-ce que cette chose qu'est la punition et qui, pendant des siècles, des millenaires peut-être, a paru à peu près évidente à la civilisation occidentale. est-ce que cette notion même de punition vous paraît maintenant aussi évidente que cela? Qu'est-ce que cela veut dire, d'être puni? Est-ce que vraiment il faut être puni?

C'est la première raison pour laquelle j'étais un peu embarrassé quand on m'a dit qu'il fallait que je parle des alternatives à la prison. Et puis j'étais embarrassé à l'idée qu'il fallait que j'en parle dans le cadre d'une semaine qui était consacrée à la faillite de la prison, parce que, et ça va peut-être vous paraître un peu paradoxal, moi, je n'ai pas du tout l'impression que la prison a fait faillite. J'ai l'impression qu'elle a parfaitement réussi.

Alors je voudrais d'abord un petit peu vous parler de ce problème de l'alternative - alternative à la prison. Je voudrais commencer par prendre comme point de repère un exemple ou une série d'exemples, qui peuvent passer à l'heure actuelle comme des tentatives de recherche d'une alternative à la prison, si vous voulez.

Je vais prendre d'abord l'exemple suédois: en 1965, la Suède s'est dotée d'une nouvelle législation pénale et la première sanction de cette législation, le premier effet, ça a été la création, ou en tout cas un programme de création, de sept grands établissement pénitentiaires, sept grands établissements tout à fait perfectionnés, avec à la fois toute la méticulosité nécessaire et toute l'indulgence aussi que l'on avait apprise. De ces sept établissements, un certain nombre ont été crés: le premier, le plus important, c'était celui de Kemela, sorte de grand édifice pénal, réalisation à l'état pur du panoptique de Bentham, en quelque sorte le rêve enchanté de la pénalité classique.

La prison de Kemela s'est ouverte, je crois, en 67 ou 68 ; en 1971 il se passe dans cette prison merveilleuse, ce qui se passe dans toutes les prisons, c'est-à-dire, primo, une grande évasion de groupe et, deuxièmement, les gens qui se sont ainsi évadés recommencent aussitôt leur vie de délinquant.

Si l'on admet que la prison sert à deux choses: premièrement à assurer de façon sûre et continue la détention des individus, vous voyez bien que 
CONTRÔLE SOCIAL

l'évasion de Kemela prouvait à l'évidence que l'appareil n'était pas au point. Deuxièmement, le fait que, à peine échappés de cette prison si merveilleusement modèle, ils aient récidivé, prouvait à l'évidence que la fonction corrective de la prison, l'objectif d'amendement qu'on nous propose à la détention, avait lui aussi échoué.

Alors que signifie une prison aussi parfaite, s’il n'y a ni sécurité ni amendement?

Les Suédois en ont immédiatement pris conscience, ou ombrage, comme vous voudrez, et, en 1973, les voilà qui ont justement essayé de définir un programme alternatif à ces prisons, à ces prisons classiques.

Programme alternatif qui consistait en quoi? En établissements, en un sens fort différent de la prison : petit établissements de 40 à 60 personnes au maximum, dans lesquels les individus étaient tenus à l'obligation du travail certes, mais un travail qui n'était pas du tout du type de travail pénal, c'est-à-dire vous savez, ce travail bête, stupide, inintéressant, abrutissant, humiliant, non payé, etc. Non, c'était du vrai travail, un vrai travail, réel, utile, payé selon les normes du travail extérieur, travail si vous voulez vous insérer dans la réalité économique du pays.

C'était des établissements aussi qui cherchaient à établir le maximum de contact entre les individus et le monde extérieur - essentiellement contact avec la famille, avec l'entourage habituel - au lieu de chercher, comme dans les prisons traditionnelles, à les rompre; dans ces établissements, on prévoit un certain nombre de chambres, une sorte de petit hôtel, de pension de famille pour l'entourage des détenus; leur famille, leur mâ̂tresse éventuellement, viendraient les voir, avec des chambres communes d'ailleurs, pour que les détenus puissent faire l'amour avec leur femme ou leur maîtresse.

Dans ces prisons également, non seulement la gestion du côté, si vous voulez, purement financier, non seulement dis-je, l'intendance de la prison mais le programme, le programme pénitentiaire lui-même est discuté, entre l'administration d'une part et puis des conseils de prisonniers qui participent à l'élaboration du régime pénal, général et commun à tous les prisonniers et éventuellement individuel, qu'il faut appliquer à chacun d'entre eux.

Enfin, dans ces prisons nouveau modèle, ou plutôt dans ces établissements alternatifs à la prison, on multiplie les permissions de sortie, et non plus simplement comme récompense pour bonne conduite, mais comme moyen de réinsertion. 
Je vous ai présenté cet exemple. J'aurais pu vous citer l'exemple allemand, c'est-à-dire des établissements qui ont été développés depuis 1970, des établissements généraux de détention de type classique, et autour desquels on a cherché à établir des institutions de type non exactement carcéral, établissements de transition et de formation professionnelle, centres sociaux et thérapeutiques comme celui de Buren, par exemple, avec des plans d'exécution pénitentiaires individualisés pour chaque détenu, des fermes, des établissements - foyers ruraux pour les jeunes délinquants.

J'aurais pu vous citer également le programme qui est celui de ce groupe dit d'anti-criminologie de Versel, Vanest, Ringelheim en Belgique, qui propose également des institutions de ce type, en particulier des établissements qui fonctionnent avec une participation directe du public, du public qui doit non seulement veiller à l'administration générale de la maison, mais également prendre en charge une sorte de jugement permanent, de consultation permanente sur les progrès des détenus et la possibilité pour eux d'avoir un travail, d'être admis en semi-liberté, d'être admis en liberté definitive, etc.

Dans tous ces établissements qui se présentent donc comme alternatifs à l'ancienne prison, de quoi s'agit-il? Eh bien! il me semble qu'en fait ces établissements, beaucoup plus que des alternatives à la prison, sont des sortes de tentatives pour essayer de faire assumer par des mécanismes, par des établissements, par des institutions qui sont différents de la prison... de faire assumer quoi?

Eh bien ! au fond, tout simplement des fonctions qui ont été jusque-là les fonctions de la prison elle-même. Essentiellement, on peut dire les choses de la manière suivante: dans toutes ces nouvelles pratiques, l'opération pénale que l'on cherche est une opération qui est centrée sur le travail; c'est-à-dire que l'on conserve, on essaie simplement de perfectionner la vieille idée, aussi vieille que le $X^{2} X^{e}$ siècle ou le $X V_{I I}{ }^{e}$ siècle, on essaie de conserver cette idée, que c'est le travail qui a en lui-même une fonction essentielle dans la transformation du prisonnier et dans l'accomplissement de la paix.

Ça serait le travail qui serait susceptible de prévenir de la manière la plus sûre les infractions. Ça serait le travail qui serait capable, mieux que tout autre chose, de punir le plus réellement. Ca serait le travail qui constituerait la vraie rétribution sociale du crime. Ça serait le travail qui serait capable, mieux que tout autre chose, de corriger l'infracteur. Autrement dit, le travail, c'est la réplique essentielle, fondamentale, à l'infraction. 
Cette idée, c'est une idée qui est à la fois classique et relativement nouvelle. Relativement nouvelle dans la mesure où c'est au XVIII ${ }^{\mathrm{e}}$ siècle, à la fin du XVIII ${ }^{\mathrm{e}}$ siècle, qu'on a eu la curieuse idée de dire qu'à une infraction il fallait répondre par autre chose que, par exemple, la mort, ou le supplice, ou l'amende ou l'exil. Qu'il fallait répondre à l'infraction par un certain travail obligatoire et contraignant, apposé à l'individu. Idée nouvelle à cette époque-là, mais idée qui depuis ce moment-là est devenue parfaitement classique, qui a d'ailleurs parfaitement échoué chaque fois qu'on a voulu l'appliquer. Mais toute la prison, toute l'organisation, tout le fonctionnement de la prison depuis le début du XIX $X^{\mathfrak{e}}$ siècle a toujours été centré autour de ce problème du travail, autour de cette idée, en tout cas, que le travail, c'est la réplique essentielle et majeure à l'infraction. Or vous voyez que cette idée, vous la retrouvez exactement identique à elle-même dans les établissements alternatifs à la prison; certes, on emploie d'autres moyens; il ne s'agit pas exactement du même travail, il n'est pas inséré exactement de la même façon dans la réalité économique de la société, mais c'est finalement toujours le travail comme réplique majeure à l'infraction que vous retrouvez dans les établissements alternatifs à la prison et dans l'ancienne prison.

Deuxièmement, je crois également qu'on voit fonctionner dans ces établissements alternatifs à la prison le principe que $\mathrm{j}$ 'appellerai le principe de refamilialisation, c'est-à-dire que vous retrouvez toujours mis en ouvre selon d'autres moyens, mais toujours mise en cuvre, l'idée que la famille, c'est l'instrument essentiel de la prévention et de la correction de la criminalité. Au XIX ${ }^{\mathfrak{e}}$ siècle, on avait déjà cette idée; on dirait maintenant qu'on employait de très curieux moyens pour refamilialiser le détenu, puisqu'on chargeait essentiellement, ou bien des aumôniers, ou bien des visiteurs de prisons, ou bien des philanthropes, on les chargeait donc par leur sermons, par leurs exhortations, par leurs bons exemples, etc. d'assurer cette refamilialisation. Refamilialisation par conséquent abstraite, fictive, etc., mais c'était elle tout de même qui était un des fils directeurs du travail pénal tel qu'on le voyait fonctionner, tel qu'on le faisait fonctionner au XIX $\mathrm{X}^{\mathrm{e}}$ siècle. Et c'est si vrai d'ailleurs que, lorsqu' on a commencé à établir des maisons de détention pour les jeunes gens, comme à Méprès, par exemple, en France, ça se passait en 1840 , le principe de refamilialisation a été appliqué avec plus de rigueur encore que dans les autres prisons. On a essayé de constituer des sortes de familles plus ou moins artificielles autour des enfants. Ensuite, on a relayé ça par des placements familiaux. De toute façon, pendant tout le XIX $\mathrm{X}^{\mathrm{e}}$ siècle, on a toujours considéré que la famille était un des agents fondamentaux de la légalité, de la vie légale ou du retour à la vie légale. 
Or cette idée que la famille doit être un agent de légalité, cette idée vous la retrouverez telle quelle, mise en ceuvre simplement par d'autres moyens, dans les établissements alternatifs à la prison.

Enfin, dans les établissement contemporains, on cherche à faire participer les détenus eux-mêmes, les conseils de détenus, etc. à l'élaboration du programme pénal. Je crois que ce qu'on fait, c'est au fond de rechercher à faire participer l'individu puni aux mécanismes mêmes de sa punition. L'idéal, ce serait que l'individu puni, soit individuellement, soit collectivement, accepte lui-même, sous la forme du conseil, la procédure de châtiment qu'on lui applique.

Et si on lui donne une part de décision dans cette définition de la peine, dans cette administration de la peine qu'il doit subir, si on lui donne une certaine part de décision, c'est bien précisément pour qu'il l'accepte, c'est bien précisément pour qu'il la fasse fonctionner lui-même; il faut qu'il devienne le gestionnaire de sa propre punition.

Or ceci est un vieux principe également, qui était le principe de l'amendement, tel qu'ont essayé de le faire fonctionner les pénalistes du $\mathrm{XIX}^{\mathrm{e}}$ siècle. Pour eux, un individu commençait à s'amender lorsqu'il était capable d'accepter sa propre punition, lorsqu'il était capable de prendre en charge sa propre culpabilité.

La mise en œuvre de cette idée à cette époque-là, surtout vers les années 1840-1850, on la cherchait plutôt du côté de l'isolement du détenu, de la vie cellulaire qui était censée l'amener au recueillement et à la réflexion. Maintenant, ce n'est plus du côté de la cellule, mais du côté du conseil de décision que l'on cherche et que l'on cherche toujours le même objectif, c'est-à-dire l'autopunition comme principe de la correction.

L'autopunition comme principe de la correction, la famille comme agent de la correction, et comme agent de la légalité, le travail comme instrument essentiel de la pénalité: ces 3 grands mécanismes qui ont caractérisé le fonctionnement de la prison tout au long du XIX $\mathrm{X}^{\mathrm{e}}$ siècle, vous les voyez fonctionner maintenant, encore et plus que jamais dans les établissements dits alternatifs à la prison. De toute façon, ce sont les vieilles fonctions carcérales que l'on essaie maintenant de faire fonctionner à partir d'établissements qui ne ressemblent plus exactement à la prison.

En un sens, on peut dire que la mise en question de la prison, sa démolition partielle, l'ouverture de certains pans de mur dans la prison, on peut dire que tout ceci libère jusqu'à un certain point le délinquant de l'enfermement strict, complet, exhaustif auquel il était voué dans les prisons du XIX siècle. 
On libère jusqu'à un certain point le délinquant, mais je dirais qu'on libère autre chose en même temps que lui; on libère peut-être quelque chose de plus que lui, on libère des fonctions carcérales. Les fonctions carcérales de resocialisation par le travail, par la famille et par l'autoculpabilisation, cette resocialisation, elle est au fond maintenant non plus localisée seulement dans le lieu fermé de la prison, mais, par ces établissements relativement ouverts, on essaie de répandre, de diffuser ces vieilles fonctions dans le corps social tout entier.

On dirait que ces établissements, qu'on cherche à définir en Suède, en Allemagne, en Belgique, en Hollande, sont de type nouveau...

Mais ces établissements ne sont peut-être pas les vraies alternatives, en tout cas les seules alternatives à la prison que l'on a conçues.

Il y en a d'autres, en effet, 'dans la mesure où - et, de plus en plus c'est vrai - on cherche une forme de pénalité qui ne passerait pas par la mise en institution des individus; qui ne les placerait, par conséquent, pas exactement, ni dans l'institution de détention classique, ni dans une maison de détention disons moderne, améliorée, alternative à la prison.

Cette punition. ce système punitif, qui ne passeraient pas par des établissements de détention, plus ou moins ouverts, ou plus ou moins fermés... eh bien ! il y en a mille exemples. Ça a commencé relativement tôt avec, si vous voulez, la généralisation de la pratique du sursis, sursis simple ou sursis avec mise à l'épreuve... Beaucoup plus récemment, dans ces dernières années, ça a été la suppression des peines de courte durée, ces suppressions que vous voyez dans des pays comme la Pologne, où, je crois, on a supprimé toutes les punitions inférieures à 3 mois; vous trouverez en Allemagne la suppression des punitions inférieures à un mois; vous trouvez également le projet, et jusqu'à un certain point, en Hollande, la mise en application d'un système de punition où le régime des amendes serait à la fois étendu, mais en même temps assoupli et rendu plus supportable, moins injuste en particulier au regard des différences de statut économique; vous avez aussi des tentatives de chercher une alternative à la prison dans la suppression d'un certain nombre de droits, comme par exemple le droit de conduire, la suppression du permis de conduire, la possibilité de se déplacer, etc. Alternative a la prison, aussi, par l'imposition d'un certain travail, mais qui se ferait en milieu ouvert, et qui ne supposerait absolument pas l'enfermement, même partiel, d'un individu.

Bon, tout ça indique qu'en effet on cherche à punir l'individu autrement que par cette espèce de prise de corps qui se fait dans une maison de 
détention; qu'il s'agisse d'une maison classique ou qu'il s'agisse d'une maison améliorée, ou d'une maison alternative à la prison.

Mais je crois que, de toute façon, même dans ces formes plus réellement alternatives à la prison dont je viens de vous parler, il faut vous faire remarquer aussitôt un certain nombre de choses. D'abord que, bien sûr, elles sont encore, et vraisemblablement elles ne peuvent être, d'une extension relativement limité ; il faut faire remarquer aussi que, pour beaucoup d'entre elles, comme par exemple le sursis, ou comme la détention partielle à mi-temps, c'est essentiellement une manière de retarder la prison, de différer le moment où le sujet y va, ou c'est en quelque sorte une manière de diluer le temps de prison sur toute une phase d'existence, et qu'en somme ce n'est pas réellement un autre système qui ne passerait pas par la détention. Et puis, et c'est là surtout le point essentiel, je crois qu' au fond ces nouvelles méthodes par lesquelles on essaie de punir des individus sans les mettre dans des prisons, ces nouvelles manières sont, elles aussi, une certaine manière de relancer, de mieux assurer en quelque sorte, les vieilles mêmes fonctions carcérales dont je vous parlais tout à l'heure.

Imposer une dette à un individu, lui supprimer un certain nombre de libertés, comme celle de se déplacer, c'est encore une fois une certaine manière de le fixer, de l'immobiliser, de le rendre dépendant, de l'épingler à une obligation de travail, une obligation de production, ou une obligation de vie de famille... c'est surtout, enfin, autant de manières de diffuser hors de la prison des fonctions de surveillance, qui vont maintenant s'exercer non plus simplement sur l'individu enfermé dans sa cellule ou enfermé dans sa prison, mais qui vont se répandre sur l'individu dans sa vie apparemment libre. Un individu en probation, eh bien ! c'est un individu qui est surveillé dans la plénitude ou dans la continuité de sa vie quotidienne, en tout cas dans ses rapports constants avec sa famille, avec son métier, avec ses fréquentations; c'est un contrôle qui va s'exercer sur son salaire, sur la manière dont il utilise ce salaire, dont il gère son budget; surveillance sur son habitat également. Bon.

Les formes de pouvoir qui étaient propres à la prison, eh bien! tout ce système alternatif à la vieille détention, toutes ces formes alternatives, elles ont pour fonction de diffuser, au fond, ces formes de pouvoir, de les diffuser comme une forme de tissu cancéreux, au-delà des murs mêmes de la prison.

C'est un véritable sur-pouvoir pénal, ou un sur-pouvoir carcéral, qui est en train de se développer, dans la mesure même où l'institution prison, elle, est en train de diminuer. Le château tombe, mais les fonctions sociales, les fonctions de surveillance, les fonctions de contrôle, les fonctions de 
resocialisation qui étaient censées être assurées par l'institution-prison, on cherche maintenant à les faire assurer par d'autres mécanismes.

Alors, bien sûr, il ne faut pas dire immédiatement: c'est bien pire, on est en train de nous préparer, avec ces soi-disant alternatives à la prison, quelque chose qui sera bien pire que la prison.

Ce n'est pas pire sans doute, mais je crois qu'il faut bien garder à l'esprit qu'il n'y a rien là qui soit véritablement alternatif par rapport à un système d'incarcération. Il s'agit bien plutôt de la démultiplication des vieilles fonctions carcérales, que la prison avait essayé d'assurer d'une manière brutale et frustre et qu'on essaie maintenant de faire fonctionner d'une manière beaucoup plus souple, beaucoup plus libre, mais aussi de manière beaucoup plus étendue. Il s'agit toujours de variations sur le même thème, de variations sur le même air; sur la même petite chanson, qui est la pénalité de détention: quelqu'un a commis une illégalité, quelqu'un a commis une infraction, eh bien ! on va s'emparer de son corps, on va le prendre en charge plus ou moins totalement, on va le mettre sous surveillance constante, on va travailler ce corps, on va lui prescrire des schémas de comportement, on va le soutenir perpétuellement par des instances de contrôle, de jugement, de rotation, d'appréciation. Tout ceci, eh bien ! c'est le vieux fond des procédés punitifs du XIX ${ }^{e}$ siècle, que vous voyez maintenant assuré sous une nouvelle forme, formes qui ne sont pas alternatives à la prison, mais dont je dirais qu'elles sont itératives par rapport à la prison. Ce sont des formes de répétition de la prison, des formes de diffusion de la prison, et non pas des formes qui sont censées la remplacer.

Et alors, maintenant, je voudrais poser le problème: il n'en reste pas moins que l'institution de prison est en train, actuellement, de subir, je ne dirais pas de toutes parts, mais sur un très large front, de subir un certain nombre de critiques, de mises en question, qui sont vraisemblablement appelées à prendre de plus en plus de diffusion.

La question que je voudrais poser maintenant est celle-ci: de deux choses, quelle est celle que l'on doit dire? On peut d'abord dire ceci: la prison apparemment disparaît, mais puisque l'essentiel des fonctions qu'elle était censée assurer sont prises en charge maintenant par de nouveaux mécanismes, au fond ça ne changerait rien. Faudrait-il plutôt dire ceci : la prison ayant disparu, eh bien! les fonctions carcérales, qui maintenant se diffusent en dehors de ses murs, ces fonctions carcérales, ne vontelles pas tout de même, petit à petit, rentrer en régression, privées qu'elles sont de leur point d'appui ; est-ce qu'elles ne vont pas disparaître? Autrement dit, est-ce que l'organe ne commence pas par disparaître, et puis finalement la fonction elle aussi s'éteindra? 
Quel sens politique faut-il donner au fait qu'actuellement, non seulement, disons dans les milieux dits d'opposition, mais également dans les conseils des différents gouvernements, dans les décisions prises par les différents États, la prison est effectivement mise en question?

Ce début de recherche d'une alternative à la prison, est-ce que c'est la disparition, et de la prison pour bientôt, et des fonctions de la prison pour plus tard?

Ou bien est-ce que c'est exactement la reconduction des mêmes mécanismes pénaux essentiels?

C'est cette question-là que je voudrais maintenant aborder; et je croís que pour l'aborder, eh bien! il faut d'abord répondre à une première interrogation: au fond, la prison, la prison sous forme consistante, la prison compacte, la prison avec ses murs, la prison avec ses cellules, la prison avec son régime disciplinaire, etc., à quoi servirait cette institution ? En quoi est-ce qu'elle était utile? Qu'est-ce qui se passait au fond? Qu'est-ce qui s'est passé pour que la prison, inventée comme moyen pénal à la fin du $\mathrm{XVIII}^{\mathrm{e}}$ siècle, ait subsisté pendant 200 ans, et subsiste encore maintenant malgré les innombrables critiques qu'on a pu en faire; et qu'on a pu en faire non pas simplement depuis ces dernières années, mais depuis la naissance même de la prison, puisque, pratiquement, il n'y avait pas 20 ans que les prisons avaient été institutionnalisées comme grand moyen de punition, les prisons n'avaient pas été plus tôt inscrites dans les codes pénaux européens que déjà on en faisait une critique radicale, qui était à peu près celle que l'on trouve formulée maintenant? À quoi servirait donc la prison?

Je crois qu'il faut répondre à cette question, si l'on veut voir, ce que peut signifier, actuellement, ce mouvement de recherche d'une mesure alternative à la prison.

Eh bien! je voudrais commencer par formuler une espece d'hypothèse, d'hypothèse-paradoxe, parce que, à la différence de l'hypothèse véritablement scientifique, je ne suis pas sûr qu'elle puisse être vérifiée avec des arguments parfaitement «complets». Je pense que c'est une hypothèse de travail, je pense que c'est une hypothèse politique, disons, si vous voulez, que c'est un jeu stratégique dont il faudrait bien voir jusqu'où elle peut nous entraîner.

Cette hypothèse, c'est celle-ci. La question serait donc celle-ci : est-ce qu'une politique pénale - c'est-à-dire d'une façon générale, Ia définition d'un certain nombre de délits et d'infractions, les règles de procédure par lesquelles on entend réagir à ces infractions, et les punitions que l'on prévoit pour elles - bref! est-ce qu'une politique pénale dans les différentes 
sociétés, a bien effectivement pour fonction, comme elle le prétend, comme on le dit, de supprimer les infractions?

Est-ce qu'au fond, une politique pénale, un code des règles de procédure, des mécanismes de punition, est-ce que tout cet appareil judiciaire, apparemment destiné à réprimer les infractions, est-ce qu'il n'est pas en fait, destiné à organiser les illégalismes? À les différencier, à établir entre eux une sorte de hiérarchie, de manière à en tolérer certains, à en punir d'autres, à en punir certains d'une manière, à punir les autres d'une autre manière?

Est-ce que la machinerie pénale n'a pas pour fonction, plutôt que de viser à l'extinction des illégalismes, de viser au contraire à leur contrôle, à leur maintien dans un certain état d'équilibre, qui serait économiquement utile et politiquement fécond? Est-ce qu'en un mot la politique pénale ne doit pas être comprise comme une certaine manière de gérer les illégalismes?

Ou encore: la pénalité, est-ce que c'est vraiment une guerre menée contre les infractions? Est-ce que ce n'est pas simplement une certaine économie concertée des infractions?

Que le système pénal ne soit pas véritablement, malgré les ordres qu'il se donne, un appareil de répression des délits, mais un mécanisme de gestion, d'intensification différentielle, de diffusion des illégalismes, de contrôle et de distribution de ces différents illégalismes, eh bien! je crois qu'on pourrait en trouver la preuve, tout simplement, dans le fonctionnement même de la prison.

On a bien souvent étudié les institutions carcêrales, on a bien décrit les aménagements architecturaux, les règlements méticuleux des prisons, etc. Tout ceci a été défini. On a aussi souvent pleuré sur la misère physique et morale de ceux qui séjournent dans les prisons.

On l'a fait, on a eu bien fait de le faire, c'est entendu. Seulement, je crois qu'il faudrait étudier - et je ne suis pas sûr que ça a été fait systématiquement, ou d'une manière très probante - il faudrait, dis-je, étudier aussi la quantité et toutes les formes d'illégalismes que la prison suscite. Bien mieux, il faudrait étudier tous les illégalismes qui sont nécessaires au fonctionnement de la prison. La prison, au fond, c'est un foyer permanent d'illégalismes intenses.

Parmi toutes les institutions qui produisent des illégalismes, qui produisent des infractions, la prison est à coup sûr la plus efficace et la plus féconde. La prison comme foyer d'illégalismes, eh bien! on en aurait 
1000 preuves. D'abord bien sûr, celles qu'on connaît, c'est-à-dire que, de la prison, on sort toujours plus délinquant qu'on était. La prison voue ceux qu'elle a recrutés à un illégalisme, qui, en général, les suivra toute leur vie: par les effets de désinsertion sociale, par l'existence, là où ça existe en effet, du casier judiciaire, par la formation de groupes de délinquants, etc.

Tout ça c'est connu. Mais je crois qu'il faut souligner aussi, que le fonctionnement interne de la prison n'est possible, que par tout un jeu, à la fois multiple et complexe, d'illégalismes. Il faut rappeler que les règlements intérieurs des prisons, sont toujours absolument contraires aux lois fondamentales qui garantissent, dans le reste de la société, les droits de l'homme. L'espace de la prison est une formidable exception du droit et à la loi. La prison, c'est un lieu de violence physique et sexuelle qui est exercée sur les détenus, par les détenus eux-mêmes et par les gardiens. C'est un lieu de carences alimentaires et c'est un lieu de frustrations sexuelles contraignantes. La prison, c'est aussi, on le sait bien, un lieu de trafic incessant, et bien sûr illégal, entre les détenus, mais aussi entre les détenus et les gardiens, entre les gardiens et le monde extérieur; trafics qui sont d'ailleurs absolument indispensables à la survie des détenus qui, sans ces trafics, n'arriveraient pas à s'en sortir, à survivre, parfois même physiquement au sens strict du terme. Indispensables aussi à la survie des gardiens qui ne supporteraient pas leur situation et leur traitement, s'ils n'avaient pas ce surplus que constitue le trafic illégal permanent, qui passe par les murs de la prison. La prison, c'est également un lieu où l'administration pratique quotidiennement l'illégalisme. Elle le pratique quotidiennement, cet illégalisme, ne serait-ce que pour couvrir aux yeux de la justice et de l'administration superrieure, d'une part, et à ceux de l'opinion en général, de l'autre, tous les illégalismes qui se produisent à l'intérieur même de la prison.

Bon, on pourrait ajouter encore que la prison, c'est un lieu dont la police se sert pour recruter son petit personnel, ses indicateurs, ses hommes de main, éventuellement ses tueurs et ses maîtres-chanteurs... Bref, chaque fois que la police a besoin de basses cuvres, où va-t-elle trouver ceux qui seront plus susceptibles de lui rendre ce service? Bien sûr, dans les prisons.

Les illégalismes, qui foisonnent autour de la prison, sont sans doute plus nombreux qui dans n'importe quelle autre institution. Tout ceci est connu, Je m'excuse de revenir sur ces truismes, mais je crois qu'il est regrettable que les sociologues, qui s'intéressent tellement au fonctionnement des institutions, n'aient pas cherché à faire en quelque sorte le diagramme de tout le réseau d'illégalismes qui entretient la vie d'une prison, qui lui permet effectivement de fonctionner - les illégalismes qui passent par la prison, mais qui permettent aussi à la prison de fonctionner. 
Il n'y a pas eu je crois de ces études systématiques, simplement des descriptions. Je vous renvoie, pour ceux qui ne le connaîtrait pas, à ce livre américain de Bruce Jackson, dont le titre en anglais est In the Life, et qui vient d'être traduit en français sous le titre En prison, je crois, et qui est fait d'interviews de détenus, de détenus du Texas en général, et qui raconte par le menu, comment se passe la vie en prison. Et en particulier, il y a sur le rôle de la sexualité, sur le rôle des infractions sexuelles, des illégalismes ou des violences sexuelles, des pages qui sont absolument éblouissantes, et qui montrent comment tout ne peut fonctionner en prison que dans la mesure où tout est illégal.

Je ne sais pas si les criminologues peuvent établir quelque chose qui serait, en quelque sorte, le taux spécifique d'illégalismes, propre à chaque institution. Il me semble qu'on devrait pouvoir chercher ça, et voir par exemple quel est le taux d'illégalismes qui est nécessaire pour qu'une école fonctionne, pour qu'une banque fonctionne, pour que l'administration des impôts fonctionne.

Chaque institution a son taux d'illégalismes nécessaire et suffisant pour exister. Mais je suis bien sûr que le taux d'illégalismes nécessaire pour que la prison fonctionne et existe, ce taux d'illégalismes est certainement le taux le plus élevé qui soit dans le tableau idéal des illégalismes fonctionnels.

La prison, c'est l'illégalisme institutionnalisé. Il ne faut jamais, par conséquent, oublier qu'au cœur de l'appareil de justice que l'Occident s'est donné sous prétexte de réprimer les illégalités, il ne faut jamais oublier qu'au cour de cet appareil de justice, destiné à faire respecter la loi, il y a une machinerie qui fonctionne à l'illégalisme permanent. La prison, c'est la chambre noire de la légalité. C'est la camera obscura de la légalité.

Eh bien ! comment se fait-il qu'une société comme la nôtre, qui se soit donné un appareil à la fois si solennel et si perfectionné pour faire respecter sa loi, comment se fait-il qu'elle ait placé au centre de cet appareil un petit mécanisme qui ne fonctionne qu'à l'illégalité et qui ne fabrique que de l'infraction, que des illégalités, que de l'illégalisme?

Eh bien! je crois qu'il y a en fait beaucoup de raisons pour que les choses se passent ainsi. Je crois qu'il y en a une, tout de même, qui est peut-être la plus importante. Ce serait celle-ci: il ne faut pas oublier qu'avant que la prison n'existe, c'est-à-dire avant qu'on n'ait choisi cette bizarre petite machinerie pour faire respecter la loi par l'illégalisme, avant donc que cette petite machinerie n'ait été inventée, à la fin du XVIII ${ }^{\mathrm{e}}$ siècle, sous l'ancien régime, les mailles du système pénal, au fond, étaient des 
mailles larges. L'illégalisme était une sorte de fonction constante et générale dans la société. À la fois par impuissance du pouvoir, et aussi parce que l'illégalisme était indispensable, au fond, à une société qui était économiquement en voie de mutation. Entre le XVI ${ }^{\mathrm{e}}$ siècle et la fin du XVII ${ }^{\mathrm{e}}$ siècle, les grandes mutations constitutives du capitalisme sont en grande partie passées par des canaux qui étaient ceux de l'illégalité, par rapport aux institutions du régime et de la société. La contrebande, la piraterie maritime, tout un jeu d'évasions fiscales, tout un jeu d'exactions fiscales aussi, ont été des voies par lesquelles le capitalisme a pu se développer. Dans cette mesure-là, on peut dire que la tolérance, la tolérance collective de la société tout entière à ses propres illégalismes, était une des conditions, non seulement de survie de cette société, mais de son développement. Et d'ailleurs, les classes sociales elles-mêmes entraient en rivalité, mais très souvent aussi en complicité, autour de ces illégalismes. La contrebande, par exemple, qui permettait à toute une couche des classes populaires de vivre, cette contrebande, elle servait non seulement à ces classes populaires, mais également à la bourgeoisie; et la bourgeoisie n'a jamais rien fait au $\mathrm{XVIII}^{\mathrm{e}}$ siècle, au XVII ${ }^{\mathrm{e}}$ siecle déjà, pour réprimer la contrebande, populaire sur le sel, le tabac, etc. L'illégalisme était un des chemins, à la fois de la vie politique et du développement économique. Or, lorsque la bourgeoisie est parvenue, non pas exactement au pouvoir au XIX siècle - elle l'avait depuis longtemps --, mais lorsqu'elle est arrivée à organiser son propre pouvoir, à se donner une technique de pouvoir qui était homogène et cohérente avec la société industrielle, il est évident que cette tolérance générale à l'illégalisme, cette tolérance générale ne pouvait plus être acceptée.

Bien sûr, la bourgeoisie a continué elle aussi, comme toute société, à fonctionner à l'illégalité. Elle s'est aménagé toute une série d'illégalismes qui lui sont profitables et qui concernent les impôts, les droits des sociétés, etc. bref, tous les grands trafics du capitalisme passent bien par une illégalité.

Mais si la bourgeoisie est parfaitement tolérante à son propre illégalisme, en revanche, les illégalismes populaires, qui lui avaient tant servi au $\mathrm{XVII}^{\mathrm{e}}$ siècle et au $\mathrm{XVIII}^{\mathrm{e}}$ siècle, ces illégalismes, maintenant, à partir du $\mathrm{XIX}^{\mathfrak{e}}$ siècle, ont cessé d'être tolérables. Et du coup, elle a cherché à réprimer et à contrôler ces illégalismes populaires, qui étaient devenus intolérables du point de vue économique, et qui étaient intolérables également pour des raisons politiques.

Au point de vue économique, en effet, à partir du moment où s'est développée une bourgeoisie capitaliste qui mettait entre les mains des ouvriers et des masses d'ouvriers des machines, des outils, des matières premières, etc., toute contrebande, tout déprédation, tout petit vol minuscule 
finissaient par prendre, par cumul, des proportions qui devenaient économiquement intolérables ou dangereuses. La moralité de l'ouvrier était absolument indispensable, dès lors qu' on avait une organisation économique de type industriel. La Révolution, et tous les troubles populaires qui, de la fin du XVIII ${ }^{\mathrm{e}}$ siècle jusqu'au milieu du $\mathrm{XIX}^{\mathrm{e}}$, ont fait trembler l'Europe, ces mouvements politiques rendaient aussi nécessaire le contrôle serré de tous les illégalismes populaires. Par conséquent, la bourgeoisie a eu besoin d'une mécanique pénale qui soit telle que la vieille tolérance, caractéristique de l'ancien régime à l'égard des illégalismes, cette tolérance disparaisse.

Et parmi les moyens qui ont été utilisés pour faire pression sur ces illégalismes populaires, pour les réduire, pour les coder et les ramener à la légalité, eh bien, il y en a un certain nombre de moyens, et l'un des moyens, ça a été précisément l'organisation, d'une certaine forme en quelque sorte privilégiée d'illégalisme, ça a été en quelque sorte la constitution d'une frange d'individus voués de façon définitive à un illégalisme professionnel, qu'on peut appeler en gros la délinquance.

En effet, avec un secteur marginal dans la population voué définitivement à la délinquance, on se donnait un formidable instrument de pouvoir. D'abord, à partir du moment où l'illégalisme est en quelque sorte professionnalisé, repris en charge par une frange déterminée de la population, on peut bien sûr le surveiller beaucoup plus facilement que lorsqu'il est diffusé à travers tout le corps social. Deuxièmement, dans la mesure où ce groupe d'illégalistes professionnels existe en lui-même, il entre en conflit avec la masse de la population, qui est évidemment la première victime des activités illégalistes du groupe. Le conflit entre les délinquants, d'une part, et la masse de la population a été un objectif perpétuellement poursuivi par le pouvoir depuis de $\mathrm{XIX}^{\mathrm{e}}$ siècle. L'existence, d'autre part, de ce groupe de délinquants, à l'égard duquel la population ne peut pas ne pas avoir des réactions d'hostilité, va rendre beaucoup plus acceptable, beaucoup plus tolérable la présence permanente de la police au milieu même de la population.

L'appel à la peur, sans cesse relancée par la littérature policière, par les journaux, par les films maintenant, l'appel à la peur du délinquant - toute la formidable mythologie apparemment glorifiante, mais en fait apeurante, cette énorme mythologie que l'on a bâtie autour du personnage du délinquant, autour du grand criminel - a rendu en quelque sorte naturelle, a naturalisé la présence de la police au milieu de la population. La police, dont il ne faut pas oublier que c'est une invention Egalement récente, et il faut bien dire, puisque je suis français, donc chauvin, que c'est à la France que l'on doit la belle instauration de la police, qui a été imitée par tous les 
pays du monde, à la fin du XVIII ${ }^{\mathrm{e}}$ siècle et au début du XIX ${ }^{\mathrm{e}}$. Enfin, ce groupe de délinquants ainsi constitué et ainsi professionnalisé, il est utilisable par le pouvoir, il est utilisable à beaucoup de fins. Il est utilisable pour des tâches de surveillance. C'est parmi ces délinquants qu'on va recruter les indicateurs, les espions, etc. Il est utilisable aussi pour tout un ensemble d'illégalismes profitables à la classe au pouvoir. Les trafics illégaux que la bourgeoisie ne veut pas faire elle-même, eh bien! elle les fera faire tout naturellement par ses délinquants. Donc, vous voyez qu'en effet beaucoup de profits économiques, beaucoup de profits politiques, et surtout la canalisation et le codage serré de la délinquance ont trouvé leur instrument dans la constitution d'une délinquance professionnelle. Il s'agissait donc de recruter des délinquants, il s'agissait d'épingler des gens à la profession et au statut de délinquant; et quel était le moyen pour recruter les délinquants, pour les maintenir dans la délinquance, et pour continuer à les surveiller indéfiniment dans leur activité de délinquants? Eh bien, cet instrument, $c^{\prime}$ est bien entendu la prison.

La prison, ça a été une fabrique de délinquants; la fabrication de la délinquance par la prison, ce n'est pas un échec de la prison, c'est sa réussite, puisqu'elle était faite pour ça. La prison permet la récidive, elle assure la constitution d'un groupe de délinquants bien professionnalisé et bien fermé sur lui-même. Par le jeu du casier judiciaire, des mesures de surveillance, par la présence des indicateurs dans le milieu des délinquants, par la connaissance détaillée que la prison permet sur ce milieu. Vous voyez que cette institution de la prison permet de garder le contrôle sur les illégalismes; en excluant par ces effets toute réinsertion sociale, elle assure que les délinquants resteront délinquants, et que, d'autre part, ils demeureront, puisqu'ils sont délinquants, sous le contrôle de la police et, si l'on veut, à sa disposition.

La prison, ce n'est donc pas l'instrument que le droit pénal s'est donné pour lutter contre les illégalismes; la prisun, elle a été un instrument pour réaménager le champ des illégalismes, pour redistribuer l'économie des illégalismes, pour produire une certaine forme d'illégalisme professionnel, la délinquance, qui allait d'une part peser sur les illégalismes populaires et les réduire, et, d'autre part, servir d'instrument à l'illégalisme de la classe au pouvoir. La prison n'est donc pas un inhibiteur de délinquance ou d'illégalisme, c'est un redistributeur d'illégalisme. Et il suffirait, pour confirmer cela, de citer un ou deux exemples.

Prenez, par exemple, le rôle de la délinquance au XIX $X^{e}$ siècle et jusqu'à ces toutes dernières années, le rôle de la délinquance dans les profits sur la sexualité. La sexualité. c'est finalement un besoin qu'il devrait être en 
quelque sorte facile de satisfaire sans avoir à payer d'une manière ou d'une autre une redevance à un système quelconque de profit. Or, l'organisation méticuleuse, dans toutes les sociétés bourgeoises du XIX ${ }^{\mathbf{e}}$ siècle, d'un système de prostitution, a permis de prélever sur le plaisir sexuel des quantités d'argent absolument fabuleuses, et dont on n'a commencé à faire le bilan qu'au cours de ces toutes dernières années. Même actuellement, dans un pays comme la France, où la prostitution a tout de même perdu de son importance relative par rapport à ce qu'elle était au XIX ${ }^{\mathrm{e}}$ siècle, la prostitution constitue encore un des plus gros chiffres d'affaires que l'on puisse établir, que l'on puisse mesurer. Au XIX ${ }^{\mathrm{e}}$ siècle, il est certain que, proportionnellement, le profit prélevé sur le plaisir sexuel des gens par le biais de la prostitution a dû être absolument colossal. Or, quels sont les employés, quels sont les préposés au prélèvement et à la surveillance de ces profits sur la sexualité ? Bien entendu, le milieu délinquant: proxénètes, maquereaux, hôteliers, etc. qui, chacun à sa manière, canalisent, vers d'ailleurs des institutions financières parfaitement honorables, canalisent un profit qu'il est inavouable de prélever tous les soirs sur le plaisir des gens.

La délinquance, ça a été, d'une façon très manifeste, un des instruments dont la bourgeoisie s'est servie pour prélever un profit illégal sur une sexualité que l'on avait rendue à cet effet illégale, et par l'intermédiaire de gens qui étaient des délinquants, c'est-à-dire de gens voués professionnellement à l'illégalisme.

On pourrait dire la même chose à propos de l'utilisation des délinquants au XIX siècle dans la lutte anti-ouvrière et dans la lutte antisyndicale. Les briseurs de grèves, les agents provocateurs, les éléments de noyautage des syndicats, ils ont été traditionnellement, pendant tout le $\mathrm{XIX}^{\mathrm{e}}$ siècle, recrutés parmi les délinquants et il ne faudrait pas faire une analyse bien profonde d'un phénomène comme la Mafia, par exemple, dans les pays d'Amérique du Nord, pour voir que la délinquance continue à jouer ce rôle économico-politique qu'on lui avait donné au XIX ${ }^{\mathrm{e}}$ siècle.

Et si la Mafia se recrute dans des voies, des chemins qui ne sont pas absolument ceux de la délinquance traditionnelle, avant toutefois que la Mafia n'existe - et dans les pays où la Mafia n'existe pas - où va-t-on trouver cette main-d'œuvre pour ces trafics illégalistes? Eh bien, on va la trouver essentiellement dans la délinquance. Donc, vous voyez, je crois, que la raison pour laquelle la prison a été si importante et pendant si longtemps, la raison pour laquelle, malgré toutes les critiques et toutes les objections qu'on a pu faire à la prison dès le début du XIX ${ }^{\mathrm{e}}$ siècle, elle a subsisté si longtemps, la raison, c'est qu'elle avait en réalité une importance, un rôle politique et économique évident. 
Alors, maintenant, il reste une question à poser, et je terminerai avec elle, c'est celle-ci : on peut montrer, je crois, comment et pourquoi la prison est utile, comment et pourquoi, en tout cas, elle l'a été. Or, nous voyons maintenant, et ceci venant d'horizons différents, nous voyons que la prison est effectivement en butte à un certain nombre de critiques et que, jusqu'à un certain point au moins, elle est en train d'entrer en régression. De quoi s'agit-il dans ce phénomène? Est-ce que c'est simplement une illusion et qu'en fait les grands mécanismes de la prison vont rester en place, et par conséquent que la délinquance va continuer à fonctionner comme elle a fonctionné jusque là? Ou est-ce que, effectivement, la prison perd de son utilité et de son rôle?

Je crois qu'un premier fait doit être bien présent à l'esprit. Il est vrai que la prison commence à entrer en régression, non seulement sous l'effet de critiques externes venant de milieux qui peuvent être plus ou moins de gauche ou plus ou moins mus par une philanthropie quelconque; je crois que si la prison entre en régression et si les gouvernements acceptent que la prison entre en régression, c'est qu'au fond le besoin en délinquants a diminué au cours des années dernières. Le pouvoir n'a plus besoin de délinquants comme il pouvait en avoir besoin jusque là. En particulier, on eprouve de moins en moins le besoin urgent d'empêcher tous ces petits illégalismes qui étaient si intolérables à la société du XIX ${ }^{\mathrm{e}}$ siècle, tous ces petits illégalismes mineurs comme, par exemple, était le vol. Autrefois, il fallait terroriser les gens devant le moindre vol. Mais maintenant, on sait pratiquer des espèces de contrôles globaux, on cherche à maintenir le vol dans un certain nombre de limites tolérables, on sait calculer ce qu'est le coût de la lutte contre le vol, et ce que coûterait le vol s'il était toléré, on sait donc établir le point optimum entre une surveillance qui empêchera le vol de franchir une certaine limite, et puis une tolérance qui permet au vol de se déployer dans les limites qui sont économiquement, moralement, et aussi politiquement favorables.

La manière dont on contrôle le vol dans les grands magasins, la manière dont on est assuré contre le vol, les mesures qui sont prises contre les chèques sans provision, ce qui se passe à propos des fraudes à la maladie, des fraudes aux assurances sociales, etc. prouvent bien qu'on a parfaitement calculé, aux instances administratives ou étatiques, ce qu'est le coût de l'illégalisme, quelles sont les formes d'illégalisme que l'on peut parfaitement tolérer et quelles sont celles qu'il faut au contraire pourchasser. Par conséquent, l'idée d'une suppression radicale, individuelle, ponctuelle des illégalismes populaires est maintenant une idée que l'on considère comme politiquement dépassée et économiquement absurde. Les petits illégalismes font partie maintenant des risques sociaux acceptables. 
Deuxièmement, je crois que la délinquance, l'existence en tout cas d'un milieu délinquant, a perdu beaucoup de son utilité économique et politique. Prenez, par exemple, ce qui se passe à propos de la sexualité dont je parlais tout à l'heure. Le profit sur la sexualité était prélevé autrefois par la prostitution. Vous savez bien maintenant qu'on a trouvé d'autre moyens, et bien plus efficaces, de prélever des profits sur la sexualité: la vente des produits pour la contraception, les thérapies sexuelles, la sexologie, la psycho-pathologie sexuelle, la psychanalyse, la pornographie, toutes ces institutions sont des manières beaucoup plus efficaces et, il faut bien le dire, beaucoup plus amusantes de prélever de l'argent sur la sexualité que l'ennuyeuse prostitution.

On pourrait dire également ceci : maintenant les grands trafics internationaux, les grands trafics d'armes, de drogue, les grands trafics sur les monnaies, échappent de plus en plus à la compétence d'un milieu de délinquants traditionnels, qui étaient sans doute de braves garçons, mais qui n'étaient sans doute pas capables, formés qu'ils étaient à la prison, de devenir les grands trafiquants internationaux dont on a besoin maintenant. Main-d'œuvre trop artisanale, main-d'œuvre trop maladroite, main-d'œuvre trop marquée aussi.

Ce sont les grands capitalistes eux-mêmes qui se chargent de gérer ces grands illégalismes. Dans cette mesure-là, aussi, on peut dire que la délinquance a perdu et ne cesse de perdre de plus en plus de son efficacité et de son intêrêt politico-économique. C'est pourquoi, sans doute, la critique que l'on fait actuellement de cette vieille institution séculaire de la prison. cette critique qui est tout aussi séculaire que la prison, eh bien! pour la première fois, elle commence à mordre réellement. Ces fameuses solutions alternatives à la prison dont je vous parlais tout à l'heure, il ne faut pas s'étonner qu'on les ait inventées maintenant. Ce n'est pas sous les coups de boutoir d'une philanthropie nouvelle, ce n'est pas à la lumière d'une criminologie récente que l'on commence maintenant à accepter parfaitement de démolir les murs des prisons, ou en tout cas de les abaisser de façon notable. Si pour la première fois la prison est entamée, ce n'est pas parce que, pour la première fois, on a reconnu ses inconvénients, mais c'est parce que, pour la première fois, ses avantages commencent à s'effacer. C'est que maintenant on n'a plus besoin d'usines à fabriquer des délinquants; mais, en revanche, on a de plus en plus besoin, à mesure même justement que le contrôle par la délinquance professionnalisée perd de son efficacité, de relayer ces contrôles par d'autres, qui sont des contrôles plus subtils, qui sont des contrôles plus fins; et c'est le contrôle par le savoir, c'est le contrôle par la psychologie, la psycho-pathologie, la psychologie sociale, la psychiatrie, la psychiatrie sociale, la criminologie, etc. 
Ce sont ces contrôles-là qui vont assurer, bien plus efficacement que cet instrument finalement grossier qu'était le couple prison-délinquance, c'est tout cela, donc, qui va assurer les contrôles sociaux. Autrement dit, l'utilité économico-politique de la délinquance disparaît. On n'a plus besoin de fabriquer des délinquants, on n'a donc plus besoin d'avoir des prisons, qui sont des machines à illégalismes et des machines à propager et à contrôler les illégalismes. Mais, en revanche, on a besoin d'instruments de contrôle qui vont se substituer au couple prison-délinquance, on a besoin d'un nouveau couple, d'une nouvelle paire, qui ne va plus être la prison et la délinquance, qui va être le contrôle et les anormaux, les contrôles portant sur les individus déviants, c'est cela qui va, avec une tout autre extension. bien sûr, et une tout autre efficacité, assurer le vieux rôle de la prison et de la délinquance.

Eh bien ! de tout ceci, qu'est-ce qu' on peut conclure? Je ne conclurai point par des propositions, puisque, vous voyez, je ne crois pas à la faillite de la prison, je crois à sa réussite, sa réussite totale jusqu'au point que nous connaissons maintenant, celui où on n'a plus besoin de délinquants; et elle n'est pas mise en faillite, elle est simplement mise en liquidation normale puisqu'on n'a plus besoin de ses profits.

Et. d'autre part, il n'y a pas d'alternatives à la prison, ou plutôt les alternatives qu'on propose à la prison sont précisément des manières de faire assurer par d'autres et sur une échelle de population beaucoup plus large les vieilles fonctions que l'on demandait au couple rustique et archaïque, «prison et délinquance».

Ceci étant dit sur l'alternative à la prison et sur la faillite de la prison, qu'est-ce qu'on peut dire pratiquement? Je terminerai sur deux ou trois considérations qui sont proprement tactiques. Je dirai ceci : premièrement, faire régresser la prison, diminuer le nombre des prisons, modifier le fonctionnement des prisons, dénoncer tous les illégalismes qui peuvent s'y produire... ce n'est pas mal, c'est même bien, c'est même nécessaire.

Mais qu'on se le dise bien, cette dénonciation de la prison, cette entreprise pour faire régresser la prison, ou lui trouver comme on dit des alternatives, ce n'est en soi ni révolutionnaire, ni contestataire, ni même progressiste. À la limite ce n'est peut-être même pas gênant à long terme pour notre système, dans la mesure où, de moins en moins, elle a besoin de délinquants, et que, par conséquent, de moins en moins elle a besoin de prisons.

Deuxièmement, il faut, je pense, aller plus loin. Faire régresser la prison, ce n'est donc ni révolutionnaire ni peut-être même progressiste; ça 
peut être, si on n'y prend pas garde, une certaine manière de faire fonctionner en quelque sorte à l'état libre les fonctions carcérales qui s'étaient jusque là exercées à l'intérieur même de la prison, et qui risquent maintenant d'être libérées elles-mêmes de la prison et reprises en charge par les instances multiples de contrôle, de surveillance, de normalisation, de resocialisation. Une critique de la prison, la recherche d'une alternative à la prison, qui ne se méfierait pas. de la manière la plus scrupuleuse, de cette rediffusion des mécanismes propres à la prison, de sa rediffusion à l'échelle du corps social, serait une entreprise politiquement nocive.

Troisièmement, la question de la prison ne peut donc pas se résoudre, et ne peut même pas se poser, dans les termes de la simple théorie pénale. Elle ne peut pas se poser non plus dans les seuls termes de la psychologie ou de la sociologie du crime. On ne peut la poser. la question de la prison, et de son rôle, et de sa disparition possible, que dans les termes d'une économie et d'une politique, si vous voulez, d'une économie politique des illégalismes.

Les questions qu'il faut poser au pouvoir ne sont pas: est-ce que oui ou non vous allez cesser de faire fonctionner des vilaines prisons, qui nous font tant de mal à l'âme? - quand nous ne sommes pas prisonniers et qu'elles ne nous font pas mal au corps. Il faut dire au pouvoir: arrêtez vos bavardages sur la loi, arrêtez vos soi-disant efforts pour faire respecter la loi, dites-nous plutôt un peu ce que vous faites avec les illégalismes? Le vrai problème est: quelles sont les différences que vous, les gens au pouvoir, vous établissez entre les différents illégalismes? Comment vous traitez les vôtres et comment vous traitez ceux des autres? À quoi vous faites servir les différents illégalismes que vous gérez? Quels profits vous tirez de ceux-ci et de ceux-là?

Ce sont ces questions-là, ces questions sur l'économie générale des illégalismes, qu'il faut poser au pouvoir, mais comme, bien sûr, il ne faut pas espérer qu'il réponde, ce sont ces questions-là qu'il faut essayer d'analyser. Et toute mise en question de la loi pénale, toute mise en question de la pénalité qui ne tiendra pas compte de ce gigantesque contexte économico-politique qu'est le fonctionnement des illégalismes dans une société, sera nécessairement une manière abstraite de poser la question.

Et, finalement, si l'on veut bien reprendre la chanson, peut-être trop entendue: pas de réforme de la prison sans la recherche d'une nouvelle société, eh bien! je dirais que, s'il faut en effet imaginer une autre société pour imaginer une autre façon de punir, je crois que, dans ce rêve que l'on doit faire d'une autre société, ce qui est essentiel, ce n'est pas d'imaginer un mode de punition qui serait particulièrement doux, acceptable ou 
efficace: il faut imaginer d'abord quelque chose de préalable, et quelque chose qu'il est sans doute beaucoup plus difficile d'inventer, mais qu'il faut chercher, en dépit de tous les exemples désastreux que l'on peut avoir sous les yeux, à droite et à gauche, dans tous les sens du mot droite et gauche, en dépit donc de cela, la question qu'il faut se poser, c'est ceci : est-ce qu'on peut effectivement concevoir une société dans laquelle le pouvoir n'ait pas besoin d'illégalismes?

Le problème, ce n'est pas l'amour des gens pour l'illégalité, le problème c'est: le besoin que le pouvoir peut avoir de posséder les illégalismes, de contrôler ces illégalismes, et d'exercer son pouvoir à travers ces illégalismes. Que cette utilisation des illégalismes se fasse par la prison ou se fasse par le "Goulag», je crois que de toute façon le problème est là: peut-il y avoir un pouvoir qui n'aime pas l'illégalisme? 